Z Rheumatol 2006 65:91-91 DOI 10.1007/s00393-006-0049-0

Online publiziert: 3. März 2006

๑) Springer Medizin Verlag 2006

E. Märker-Hermann · A. Radbruch

\title{
Forschungsförderung in der Deutschen Gesellschaft für Rheuma- tologie und Zukunftsperspektiven für nationale und europäische Rheumaforschungsprogramme
}

le Mitglieder des Komitees die Anträge unabhängig voneinander, einzeln und schriftlich bewertet. Aus allen Bewertungen wurde ein Ranking erstellt. Leider erreichte keiner der eingereichten Anträge im Mittel eine gute Bewertung (eingeschränkt förderwürdig) oder besser. Das Koordinationskomitee hat dies sehr bedauert und sieht die Ursache auch darin, dass es in der Ausschreibung offenbar nicht ausreichend gelungen ist, die Kriterien für die "Start-up“Projekte deutlich zu vermitteln. Nach ausführlicher Diskussion wurde dem Vorstand der DGRh empfohlen, die Projektförderung zurückzustellen, da keines der Projekte hinsichtlich der geforderten Kriterien zu überzeugen vermochte. Der Vorstand der DGRh ist dieser Empfehlung gefolgt. In dieser Ausgabe der Zeitschrift für Rheumatologie finden Sie nun die neue Projektausschreibung 2006 für „Start-up“-Projekte Rheumaforschung. Neben einer klareren Formulierung der Voraussetzungen für Antragsteller und zur Art der zu fördernden Projekte wurde Wert darauf gelegt, dass die eingereichten Anträge vergleichend von externen (ausländischen) Gutachtern begutachtet werden. Daher sollen präzise (maximal 3 Seiten) formulierte Anträge in englischer Sprache eingereicht werden. Zusätzlich wird eine laienverständliche Zusammenfassung in Deutsch erwartet, die den Mitgliedern des Patientenbeirats im KNR vorgelegt wird.

Konkrete Förderung innovativer Forschungsprojekte, Erhalt und Ausbau der bestehenden Netzwerkstrukturen und Motivation des rheumatologischen und biomedizinischen Nachwuchses - dies sind unabdingbare Voraussetzungen dafür, dass Rheumaforschung in den zukünftigen langfristigen Förderplänen der Bundesregierung und der Europäischen Union verankert wird. Gemeinsam mit den medizinischen Nachbardisziplinen Orthopädie, Unfallchirurgie, Endokrinologie/Osteologie und Entwicklungsbiologie sollte es uns gelingen, die muskulo-skelettalen Erkrankungen in den Focus der nationalen und internationalen Forschungsförderung zu rücken.

Prof. Dr. med. Elisabeth Märker-Hermann, Präsidentin der DGRh

Prof. Dr. rer. nat. Andreas Radbruch, 1. Vizepräsident der DGRh 